\title{
Assessment of the capacity of cerebral collateral circulation using ultrasonic Doppler technique
}

\author{
KUNIHIKO TADA, TADAATSU NUKADA, SHOTARO YONEDA, \\ YOSHIHIRO KURIYAMA, AND HIROSHI ABE \\ From the Division of Vascular Disease, First Department of Internal Medicine, \\ Osaka University Medical School, Fukushimaku, Osaka, 553, Japan
}

SYNOPSIS A new non-invasive method for the assessment of cerebral collateral circulation via the circle of Willis using an ultrasonic directional Doppler flowmeter is described. The technique was found useful in measuring the capacity of cerebral collateral circulation and would be applicable not only in internal medicine but also in neurosurgery.

When one internal carotid or vertebral artery is occluded the blood flow of the remaining cranial arteries immediately increases because of interarterial shunting through the circle of Willis (Kristiansen and Krog, 1962; Symon et al., 1963; Toole, 1966). This haemodynamic response is considered as a certain circulatory reserve of the brain. The efficiency and the adequacy of emergent haemodynamic change are supposed to be important factors in determining the occurence of neurological deficit after occlusion of one or more of the aortocranial arteries (Fisher, 1954; Kristiansen and Krog, 1962).

Methods such as electroencephalography (EEG) (Toole and Bevilacqua, 1963; Meyer et al., 1965), measurement of internal carotid artery back pressure (ICA pressure) (Moore and Hall, 1969), cerebral blood flow measurement (CBF) (Jennett et al., 1966; Boysen, 1971), and the combined measurement of EEG, ICA pressure, and CBF (Trojaborg and Boysen, 1973; Leech et al., 1974) have been applied to assess the potential of collateral circulation among the aortocranial arteries via the circle of Willis. The present study was performed to evaluate a new non-invasive technique, Doppler flowmetry, for the assessment of the capacity of cerebral collateral circulation.

\section{METHODS}

BLOOD FLOW DETECTION BY DOPPLER FLOWMETERO Phasic bidirectional blood flow velocity waş $\infty$ measured using a model EUD-3B ultrasonici Doppler blood flowmeter (Hitachi Medical Corpora tion, 1-1-14, Uchikanda, Chiyodaku, Tokyo, Japan) which was connected to a Doppler probe (5 MHz응 $\frac{\Phi}{3}$ $5 \mathrm{~mm}$ in diameter). The block diagram of the Doppler blood flowmeter is shown in Fig. 1. Modeक्ष EUD-3B involves two filters for separating the received signals into those corresponding to the toward-probe flow and away-from-probe flow, respectively. The signals from 5000 to $5007 \mathrm{kHz}$ in transmitting frequency pass one of the two filters and those from 4993 to $5000 \mathrm{kHz}$ pass the other filter. Each filter has a steep frequency curve for discrimination of toward-probe and away-fromprobe directions even with low-velocity blood flow. In addition, EUD-3B uses a zero-crossing counter composed of three circuits; Schmitt trigger, monostable multivibrator, and filter. It gives a voltage output proportional to the input frequency. The output voltages from the Doppler flowmeter are connected to d.c. amplifiers inserted in a polygraph (ECP-1, Hitachi Medical Corporation) and to an electric integrator which is employed to simplify the measurement of the change of blood flow (Fig. 2).

The theoretical basis for using an electric integrator is as follows. The Doppler flowmeter measures the average velocity (in the sense of the average across the vessel) in the line of the sound beam. The average velocity (vel) across the tube is given by dividing the volume flow $(F)$ by the cross-sectional 1068 


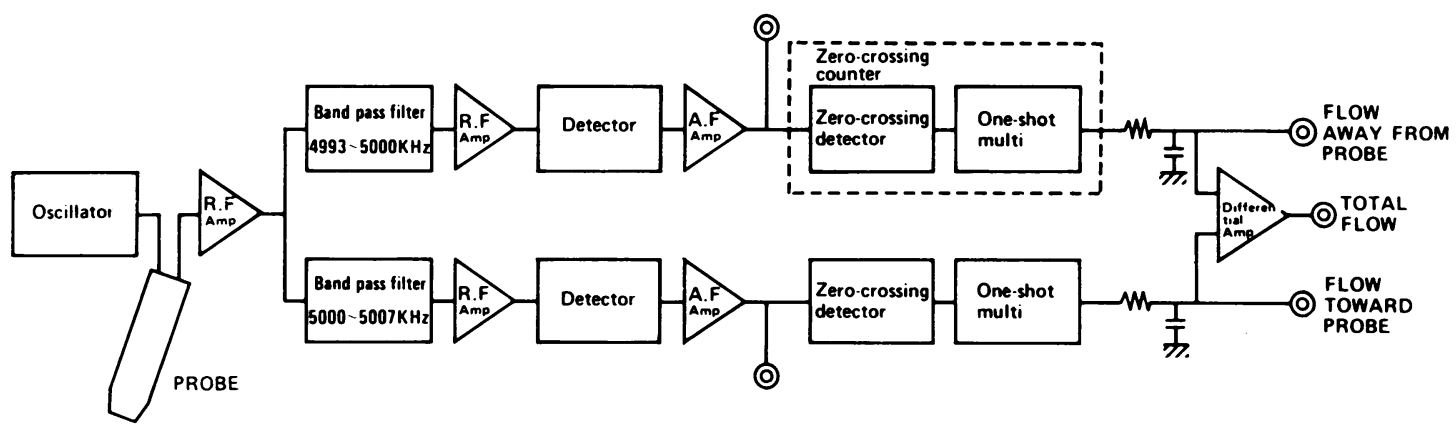

FIG. 1 Block diagram of the Doppler flowmeter.

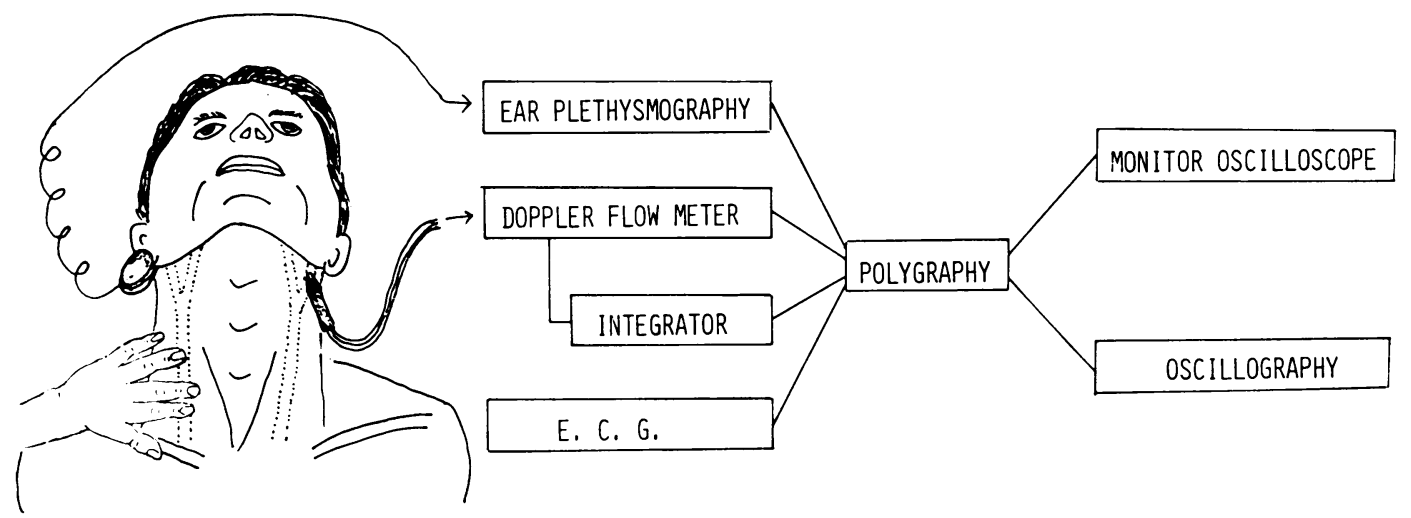

FIG. 2 Schematic layout of Doppler ultrasonic system employed in this study.

area $\left(\pi r^{2}\right)$. The volume flow through a vessel per unit time $(\mathrm{t})$ can be, therefore, expressed in accordance with the following formula.

$$
\mathrm{F}=\pi \mathrm{r}^{2} \cdot \int_{0}^{t} \text { vel } \mathrm{dt}
$$

If the calibre of the artery is constant in spite of haemodynamic change, the blood flow change of each artery can be obtained by contrasting the integrated value of velocity signal during a certain load with that before a load.

The integration of Doppler velocity signals was made automatically every three seconds by the electric integrator. The integrated value was expressed as a height of the ordinate as shown in Fig. 3. The Doppler flow profile and its integrated value, together with electrocardiogram (ECG) and ear plethysmogram, were simultaneously monitored on an oscilloscope and recorded on an oscillographic recorder (Fig. 2).

CAROTID COMPRESSION Digital carotid compression was chosen to simulate an acute occlusion of the carotid artery. To avoid a carotid sinus reflex, the carotid compression was done as far below the carotid sinus as possible.

When carotid compression was performed completely the wave of the ipsilateral ear plethysmogram was altered to a low transmission plateau wave (Fig. 3). The completeness of carotid compression, therefore, was judged by monitoring the ear plethysmogram.

To check the effects of carotid compression on systemic and clinical states, a graded carotid compression was made before full carotid compression. Clinical alternations were avoided by prompt termination of carotid compression when any clinical signs of cerebral ischaemia appeared.

INTERNAL CAROTID FLOW DETECTION The subject was in the supine position and the Doppler probe was directed to the internal carotid artery. The probe was positioned manually so as to procure maximum audible audio-signals as heard from a loudspeaker. The definition of the signal from the internal carotid 


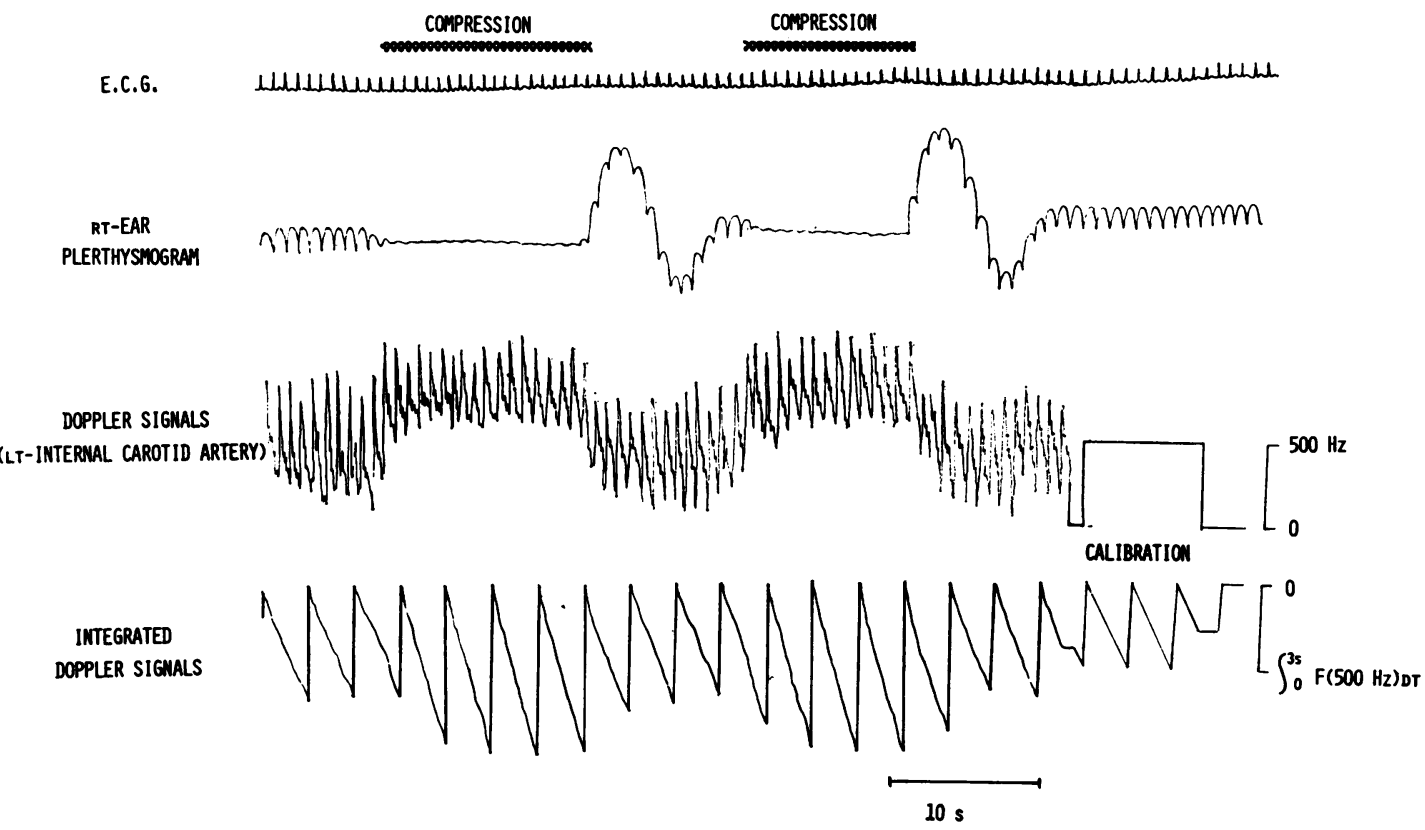

FIG. 3 A polygraphic record monitoring electrocardiogram (ECG), ear plethysmogram, Doppler signal of $\stackrel{0}{\overrightarrow{0}} \vec{\circ}$ internal carotid artery, and its electrically integrated value. This showed that digital carotid compression of the right carotid artery produced a remarkable increase in both Doppler signal and its integrated value and reduction of wave amplitude of the right ear plethysmogram. $R-R$ intervals of ECG did not change. Reproducibility of the above haemodynamic response was also demonstrated.

artery was ascertained by a test compression of the contralateral carotid artery, which resulted in consequential increase in the flow velocity signal. The signal from the external carotid artery was not influenced. The common flow direction detected by this technique was away-from-probe.

VERTEBRAL FLOW DETECTION The subject was placed supine on a firm table. Thin pads were set under both shoulders. The head was tilted back and toward the side opposite to the examination. The vertebral artery flow was detected by placing the probe at the posterior-inferior margin of the mastoid process with the beam directed medially in a moderately superior direction. The signal of vertebral flow was assured by ipsilateral carotid compression which caused increase in velocity signals. The vertebral artery was examined at the point just before it entered the foramen magnum. The Doppler profiles detected by this technique revealed an away-fromprobe flow in normal man.

GeNeral PROCEDURE After applying the Doppler probe to one internal carotid artery, contralateral carotid compression was performed for approximately 10 seconds and repeated two or three times. Before, during, and after the carotid compression the Doppler velocity profile and its integrated value were recorded continuously. The same examination was carried out on the opposite internal carotid artery.

After satisfactory examination on both internal carotid arteries, the Doppler probe was settled for the detection of vertebral flow. Ipsilateral carotid compression was followed by contralateral carotid compression. The blood velocity profile and its integrated value were recorded throughout the manipulation.

The electrocardiogram (ECG) and the ear plethysmogram of the same side as the carotid compression were usually monitored on an oscilloscope. The systemic blood pressure was measured immediately before, during and after carotid compression. The subjects were observed closely during the entire procedure.

PER CENT INCREASE IN BLOOD FLOW Per cent increase in blood flow through each aortocranial artery was 
calculated to assess the effect of one carotid artery compression on the blood flow through the remaining arteries.

$$
\text { per cent increase }=\frac{\mathrm{Fa}-\mathrm{Fb}}{\mathrm{Fb}} \cdot 100
$$

where: $\mathrm{Fa}=$ integrated value of blood velocity during carotid compression

$\mathrm{Fb}=$ integrated value of blood velocity before carotid compression

In practice, per cent increase in blood flow was easily calculated on the oscillograph paper by measuring the ordinate value which indicated the integrated value of blood velocity.

In this study, the average value of per cent increases of the left and the right carotid arteries was employed as the per cent increase of carotid artery. With regard to the vertebral artery, the average value of per cent increases of both sides during ipsilateral carotid compression was taken.

\section{Clinical application}

SUBJECTS The per cent increase in blood flow during carotid compression might be influenced by change in the systemic blood pressure and heart rate. Subjects who showed a considerable change in blood pressure and heart rate when compared before and during carotid compression were excluded from the present study. Subjects with considerably different per cent increases between the left and the right sides were also omitted.

The changes in blood flow of aortocranial arteries in $\mathbf{3 0}$ subjects were suitable for recording throughout the period of digital carotid compression. There was no complication attributable to carotid compression. The subjects were classed into three groups. The first group, represented as young healthy, consisted of 10 young healthy subjects whose ages were 15 to 35 years, mean age 26.7 years $(S D \pm 5.6)$. The second 10 subjects, represented as hypertension, had definite hypertension (systolic blood pressure above 160 $\mathrm{mmHg}$ and/or diastolic pressure above $90 \mathrm{mmHg}$ ). Their ages ranged from 34 to 65 years, mean age 50.3 years $(S D \pm 10.7)$. No subjects in this group had clinical symptoms of cerebrovascular disease. The third 10 subjects, represented as cerebrovascular disease (CVD), had been suffering from cerebrovascular diseases. Ages of the CVD group varied from 48 to 70 years, the mean being 61.7 years (SD \pm 6.6). None of the subjects was acutely ill at the time of the study.

Average age of the young healthy group was significantly younger than that of the hypertension group and that of the CVD group with both $\mathbf{P}<$ 0.001 . There was no significant difference in average age between hypertension and CVD groups.

\section{RESULTS}

HAEMODYNAMIC RESPONSE EVOKED BY CAROTID COMPRESSION When one carotid artery was compressed there was commonly an increase in blood flow through the remaining arteries. This haemodynamic response was immediate and did not change perceptibly over the usual period (approximately 10 seconds) of the carotid compression. After release of carotid compression the blood flow through the remaining arteries returned promptly to the previous level. Figure 3 shows an example of the record. The overall results of the present study are summarized in the Table.

Internal carotid artery The per cent increase in blood flow through the opposite internal carotid artery induced by digital compression of one common carotid artery varied from subject to subject from 4 to $58 \%$. The per cent increase in internal carotid artery flow in young healthy,

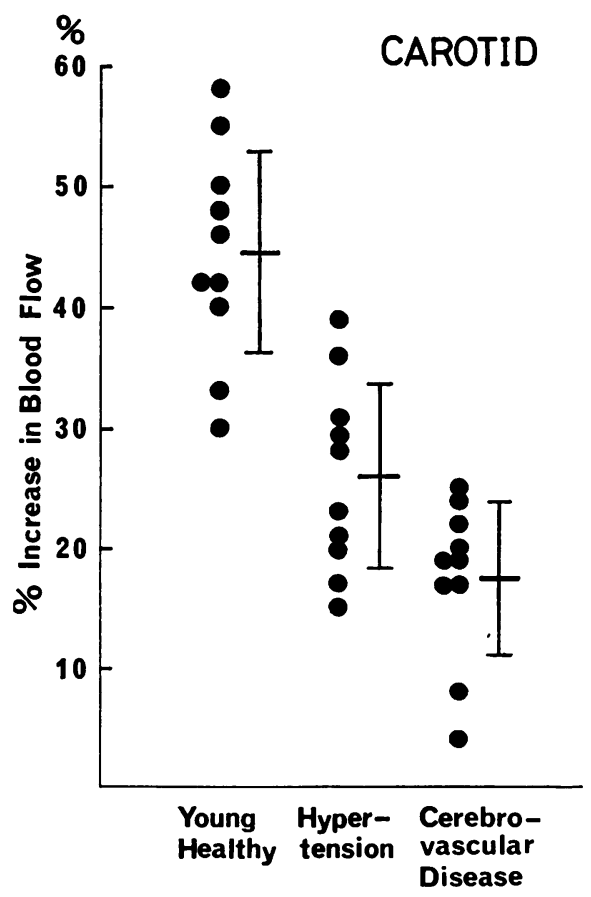

FIG. 4 Per cent increase in carotid flow during carotid compression in three groups, showing each per cent increase and the mean $\pm S D$. 


\section{TABLE}

OVERALL RESULTS IN YOUNG HEALTHY, HYPERTENSION, AND CEREBROVASCULAR DISEASE GROUPS, SHOWING AGE, MEAN ARTERIAL BLOOD PRESSURE, AND PER CENT INCREASE IN CAROTID AND VERTEBRAL BLOOD FLOW DURING CAROTID COMPRESSION

\begin{tabular}{|c|c|c|c|c|c|}
\hline & \multirow{2}{*}{$\begin{array}{l}\text { Age } \\
(y r)\end{array}$} & \multirow{2}{*}{$\begin{array}{l}\text { Mean arterial } \\
\text { blood pressure } \\
\quad(\mathrm{mmHg})\end{array}$} & \multicolumn{3}{|c|}{ Per cent increase in blood flow } \\
\hline & & & Carotid & $(\%)$ & Vertebral \\
\hline $\begin{array}{l}\text { Young healthy }(\mathrm{N}=10) \\
\text { Hypertension }(\mathrm{N}=10) \\
\text { CVD }(\mathrm{N}=10)\end{array}$ & $\begin{array}{l}26.7 \pm 5.6 \\
50.3 \pm 10.7^{*} \\
61.7 \pm 6.6^{*}\end{array}$ & $\begin{array}{c}87.0 \pm 7.9 \\
116.1 \pm 7.7^{*} \\
107.3 \pm 14.4 \dagger\end{array}$ & $\begin{array}{l}44.4 \pm 8.4 \\
25.9 \pm 7.6^{*} \\
17.5 \pm 6.3 \S\end{array}$ & & $\begin{array}{l}42.9 \pm 6.5 \\
25.9 \pm 6.5^{*} \\
17.8 \pm 5.0 \ddagger\end{array}$ \\
\hline
\end{tabular}

CVD: cerebrovascular disease.

Values are mean \pm SD.

Significantly different from young healthy: $* \mathrm{P}<0.001 ; \dagger \mathrm{P}<0.01$.

Significantly different from hypertension: $\ddagger P<0.01 ; \S P<0.05$.

hypertension and CVD groups are shown in Fig. 4. The young healthy group showed a high value of the per cent increase in internal carotid flow which ranged from 30 to 58 per cent, the mean value being $44.4 \%(\mathrm{SD} \pm 8.4)$. The hypertension group showed 15 to $39 \%$ increase in carotid flow and the mean value was $25.9 \%(\mathrm{SD} \pm 7.6)$. In the cases with CVD the carotid compression gave rise to a low per cent increase which ranged from 4 to $25 \%$, the mean being $17.5 \%$ (SD \pm 6.3$)$.

Student's $t$ test of paired differences applied to the per cent increase in young healthy and hypertension groups indicated a significant difference $(P<0.001)$. Between the per cent increase of young healthy and CVD groups there was also a significant difference $(P<0.001)$. In the $C V D$ cases the per cent increase in carotid flow showed a significantly lower value than that in hypertension $(P<0.05)$.

Vertebral artery The per cent increase in blood flow through a vertebral artery during carotid compression varied from 9 to $53 \%$, as shown in Fig. 5. The mean values of the per cent increases in vertebral flow in young healthy, hypertension and CVD groups were 42.9 (SD \pm 6.5 ), 25.9 $(S D \pm 6.5)$, and $17.8(S D \pm 5.0)$ per cent, respectively. In each group the variation of the per cent increase in vertebral flow showed the same trend as those in carotid flow (Figs 4 and 5).

There was a statistical difference in the per cent increase between young healthy and hypertension groups $(P<0.001)$. A significant difference in the per cent increase in vertebral flow was also noticed between young healthy and CVD groups $(P<0.001)$. The per cent increase in vertebral flow was greater in the hypertension group than in the CVD group, reaching the level of statistical significance $(\mathrm{P}<0.01)$.

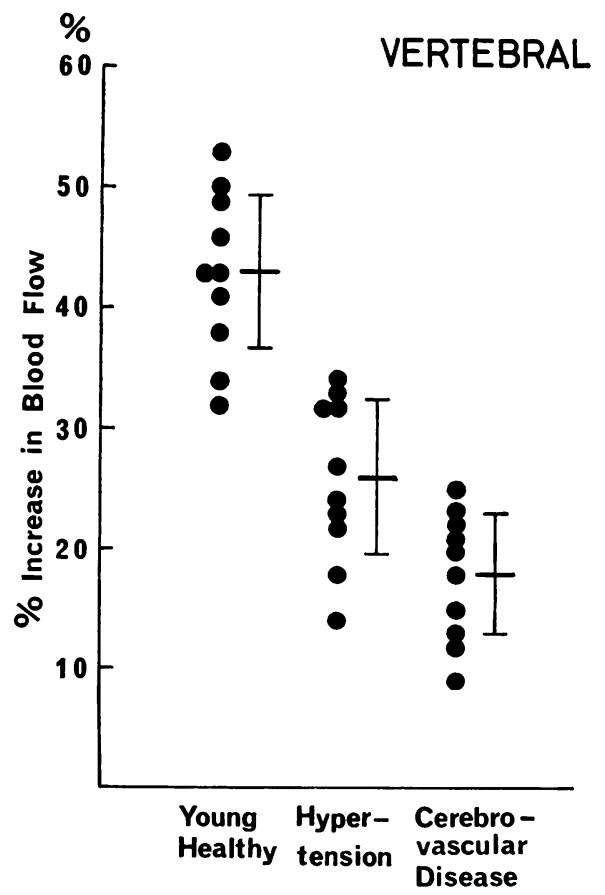

FIG. 5 Per cent increase in vertebral flow during carotid compression in three groups. To show each per cent increase and the mean $\pm S D$. 


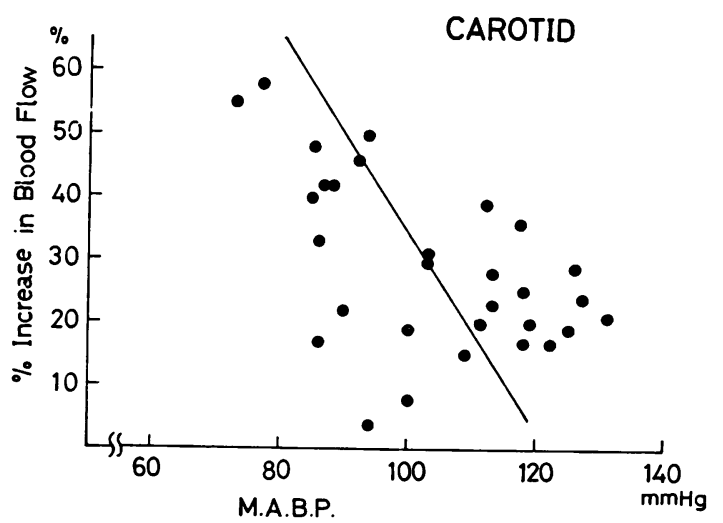

FIG. 6 A linear correlation between the per cent increase in carotid blood flow and the value of mean arterial blood pressure (MABP). $y=-1.55 x+189$; $\mathrm{r}=-0.543 ; P<0.01$.

MEAN ARTERIAL BLOOD PRESSURE AND PER CENT INCREASE The average values of mean arterial blood pressure (MABP) in young healthy, hypertension, and CVD groups were $87.0(\mathrm{SD} \pm$ 7.9), 116.1 (SD \pm 7.7$)$ and 107.3 $(\mathrm{SD} \pm 14.4)$ $\mathrm{mmHg}$, respectively. MABP was lower in the young healthy group than both hypertension and CVD groups $(\mathrm{P}<0.001$ from hypertension; $\mathbf{P}<0.01$ from CVD). MABP of the hypertension group was higher than that of the CVD group, but this did not reach the level of statistical significance.

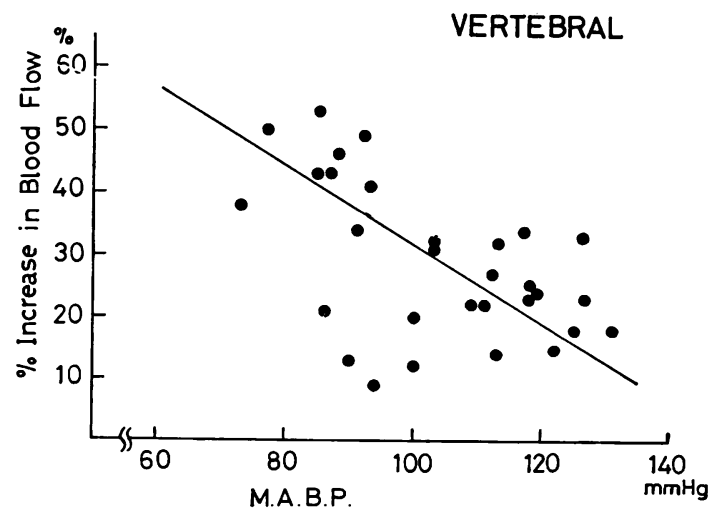

FIG. 7 A linear correlation between the per cent increase in vertebral blood flow and the value of mean arterial blood pressure (MABP). $y=-0.633 x+95$; $\mathrm{r}=-0.863 ; P<0.001$.
The relationship between the haemodynamic response of aortocranial arteries produced by carotid compression and MABP value was investigated without relation to the above individual groups. There was a linear correlation between the per cent increase in carotid flow provoked by carotid compression and the value of MABP. As represented in Fig. 6, the higher the MABP value, the lower the per cent increase $(\mathrm{P}<0.01 ; \mathrm{r}=-0.543)$. A significant linear correlation was also obtained between the per cent increase in vertebral flow and the value of $\operatorname{MABP}(\mathrm{P}<0.001 ; \mathrm{r}=-0.863)$ (Fig. 7).

\section{DISCUSSION}

In order to accommodate the brain function to various physiological and pathophysiological challenges, interarterial shunts among four aortocranial arteries through the circle of Willis play an important role in cerebral collateral circulation (Toole, 1966). The clinical and pathological data of the cases with carotid occlusion have indicated that the pathological change in the brain depends on various factors such as the patency of the opposite carotid artery, the efficacy of collateral circulation and the size of the component vessels of the circle of Willis (Fisher, 1954).

Carotid artery ligation in the neck is an accepted method of treating certain intracranial aneurysms (Nishioka, 1966) and temporary clamping of the carotid artery is an indispensable technique in the operative treatment for carotid occlusive disease (Bland et al., 1970). Carotid ligation or temporary clamping carries a risk of producing ischaemia of the ipsilateral hemisphere (Nishioka, 1966; Bland et al., 1970). Some methods have been applied for predicting the likelihood of cerebral ischaemia after carotid occlusion but a reliable method has been elusive.

Under electroencephalographic control, digital compression of a carotid artery in the neck has been performed to assess the efficiency of the cross-circulation (Toole and Bevilacqua, 1963; Meyer et al., 1965). EEG recording can reveal only indirect qualitative information regarding cerebral collateral circulation.

Measurement of internal carotid artery back pressure (ICA pressure) during temporary occlusion of a carotid artery has been used to 
determine the degree and adequacy of cerebral collateral circulation (Moore and Hall, 1969; Trojaborg and Boysen, 1973). It has been suggested that ICA pressure is of value in predicting tolerance or intolerance for temporary carotid occlusion (Moore and Hall, 1969). ICA pressure, however, could be measured only during carotid surgery.

Electromagnetic flowmetry has been used to yield quantitative information about the blood supply through the main arterial channels (Kristiansen and Krog, 1962; Symon et al., 1963; Nornes, 1973). In the monkey, Symon et al. (1963) showed that occlusion of one carotid artery produced an immediate increase in blood flow through the remaining arteries, through the opposite carotid artery of $42.5 \pm 10 \%$ and through each vertebral artery of $33.7 \pm 4.4 \%$. In the human, Nornes (1973) found a flow increase through the contralateral internal carotid artery varying between 14 and $73 \%$ at trial occlusion of the ipsilateral internal carotid artery for treating intracranial aneurysms. It was suggested that a contralateral flow increase in excess of $40-50 \%$ of control would indicate sufficient collateral capacity and that low flow increase (14-17\% of control) should be considered as a sign of poor collateral circulation. Although electromagnetic flowmetry could clarify quantitative data regarding cerebral haemodynamics, it required a surgical approach.

By use of a radioactive isotope clearance method, cerebral blood flow has been measured for the purpose of examination of the effects of carotid occlusion on cerebral circulation (Jennett et al., 1966; Boysen, 1971). It was reported that the mean value of regional cerebral blood flow (rCBF) was decreased from 51 to $30 \mathrm{ml} / 100 \mathrm{~g}$ brain $/ \mathrm{min}$, a reduction of $41 \%$, when the internal carotid artery was clamped during carotid endarterectomy (Boysen, 1971). The CBF measurement could give the researcher information regarding tissue perfusion flow, but could not give direct knowledge related to the haemodynamic responsibility of different aortocranial arteries. Since rCBF measurement requires carotid puncture and a radioisotope detector system, it cannot be carried out easily and repeatedly.

Combined measurement of EEG, ICA pressure and CBF has been carried out to obtain more detailed information concerning the haemodynamic changes during carotid occlusion and to evaluate the reliability of the different methods in the examination of cerebral collateral circulation (Trojaborg and Boysen, 1973; Leech et al., 1974). These reports have been based on observations in patients with cerebral aneurysm or carotid occlusive disease undergoing carotid surgery. There are, therefore, many stresses on the subject in applying the combined method to the field of internal medicine.

In this paper, a new method for assessing cerebral collateral circulation through the circle of Willis at the level of aortocranial arteries has been demonstrated. Transcutaneous Doppler technique is non-invasive, simple, and easy, involves little risk, and permits repeated examination at any time during days and years. Data obtained using this method are quantitative and reproducible. Since the Doppler flowmetry utilized in this study used a zero-crossing counter, there was an advantage over sonography in real time display. False results due to inadequate carotid compression, which has been considered as a disadvantage, could be prevented by use of ear plethysmogram to indicate the completeness of carotid compression. The present method made it possible to determine the blood flow not only through the carotid arteries but also through the vertebral arteries. The usefulness of transcutaneous Doppler flow detection technique for the purpose of clarification of the presence of stenosis, occlusion, reverse flow, and presence of various collateral channels has been demonstrated in the application to arterial occlusive disease in the neck (Maroon et al., 1970; Müller, 1972; Spencer et al., 1974) and to cases of brain death (Yoneda et al., 1974).

The per cent increase in flow through the contralateral internal carotid artery and both vertebral arteries during carotid compression was in good agreement with that of previous reports by use of an electromagnetic flowmeter (Symon et al., 1963; Nornes, 1973). CVD and hypertension groups showed a lower per cent increase than the young healthy group in this study. The hypertension group was shown to have a significantly higher per cent increase than the CVD group. The present study has also pointed out that there is a linear correlation between the per cent increase in both internal carotid and 
vertebral artery flows and the value of MABP. Skillicorn and Aird (1954) made clear that EEG abnormalities during carotid compression occurred more frequently in older patients than in the young. Meyer et al. (1965) suggested that the capacity of cerebral collateral circulation appeared to decrease with advancing age and with cerebrovascular disease. It has been reported that significantly greater atherosclerotic changes are noted in the cervical or cerebral arteries of patients with cerebral stroke (Martin et al., 1960; Kameyama and Okinaka, 1963) and hypertension (Young et al., 1960) and that hypertensive subjects have increased cerebrovascular resistance (Kety et al., 1948). It is, therefore, assumed that the lower per cent increase in blood flow during carotid compression in the cases with cerebrovascular disease and/or hypertension is due to atherosclerotic lesions in the cervical or cerebral arteries and to increased cerebrovascular resistance. The presence of a linear correlation between the per cent increase in blood flow and the value of MABP supports this assumption.

\section{REFERENCES}

Bland, J. E., Chapman, R. D., and Wylie, E. J. (1970) Neurological complications of carotid artery surgery. Annals of Surgery, 171, 459-464.

Boysen, G. (1971). Cerebral blood flow measurement as a safeguard during carotid endarterectomy. Stroke, 2, 1-10.

Fisher, M. (1954). Occlusion of the carotid arteries: further experiences. Archives of Neurology and Psychiatry, 72, 187-204.

Jennett, W. B., Harper, A. M., and Gillespie, F. C. (1966). Measurement of regional cerebral blood-flow during carotid ligation. Lancet, 2, 1162-1163.

Kameyama, M., and Okinaka, S. (1963). Collateral circulation of the brain with special reference to atherosclerosis of the major cervical and cerebral arteries. Neurology (Minneap.), 13, 279-286.

Kety, S. S., Hafkenschiel, J. H., Jeffers, W. A., Leopord, I. H., and Shenkin, H. A. (1948). The blood flow vascular resistance, and oxygen consumption of the brain in essential hypertension. Journal of Clinical Investigation, 27, 511-514.

Kristiansen, K., and Krog, J. (1962). Electromagnetic studies on the blood flow through the carotid system in man. Neurology (Minneap.), 12, 20-22.
Leech, P. J., Miller, J. D., Fitch, W., and Barker, J. (1974). Cerebral blood flow, internal carotid artery pressure, and the EEG as a guide to the safety of carotid ligation. Journal of Neurology, Neurosurgery, and Psychiatry, 27, 854-862.

Maroon, J. C., Campbell, R. L., and Dyken, M. L. (1970). Internal carotid artery occlusion diagnosed by Doppler ultrasound. Stroke, 1, 122-127.

Martin, M. J., Whisnant, J. P., and Sayre, G. P. (1960). Occlusive vascular disease in the extracranial cerebral circulation. Archives of Neurology, 3, 530-538.

Meyer, J. S., Gotoh, F., and Favale, E. (1965). Effects of carotid compression on cerebral metabolism and electroencephalogram. Electroencephalography and Clinical Neurophysiology, 19, 362-376.

Moore, W. S., and Hall, A. D. (1969). Carotid artery back pressure: a test of cerebral tolerance to temporary carotid occlusion. Archives of Surgery, 99, 702-710.

Müller, H. R. (1972). The diagnosis of internal carotid artery occlusion by directional Doppler sonography of the ophthalmic artery. Neurology (Minneap.), 22, 816-823.

Nishioka, H. (1966). Report on the cooperative study of intracranial aneurysms and subarachnoid haemorrhage. Section 8, part I. Results of the treatment of intracranial aneurysms by occlusion of the carotid artery in the neck. Journal of Neurosurgery, 25, 660-682.

Nornes, H. (1973). The role of the circle of Willis in graded occlusion of the internal carotid artery in man. Acta Neurochirurgica, 28, 165-177.

Skillicorn, S. A., and Aird, R. B. (1954). Electroencephalographic changes resulting from carotid artery compression. Archives of Neurology and Psychiatry, 71, 367-376.

Spencer, M. P., Reid, J. M., Davis, D. L., and Paulson, P. S. (1974). Cervical carotid imaging with a continuous-wave Doppler flowmeter. Stroke, 5, 145-154.

Symon, L., Ishikawa, S., Lavy, S., and Meyer, J. S. (1963). Quantitative measurement of cephalic blood flow in the monkey. A study of vascular occlusion in the neck using electromagnetic flowmeters. Journal of Neurosurgery, 20, 199-218.

Toole, J. F. (1966). Interarterial shunts in the cerebral circulation. Circulation, 33, 474-483.

Toole, J. F., and Bevilacqua, J. E. (1963). The carotid compression test: evaluation of the diagnostic reliability and prognostic significance. Neurology (Minneap.), 13, 601-606.

Trojaborg, W., and Boysen, G. (1973). Relation between EEG, regional cerebral blood flow and internal carotid artery pressure during carotid endarterectomy. Electroencephalography and Clinical Neurophysiology, 34, 61-69.

Yoneda, S., Nishimoto, A., Nukada, T., Kuriyama, Y., Katsurada, K., and Abe, H. (1974). To-and-fro movement and external escape of carotid arterial blood in brain death cases. A Doppler ultrasonic study. Stroke, 5, 707-713.

Young, W., Gofman, J. W., Tandy, R., Malamud, N., and Waters, E. S. G. (1960). The quantitation of atherosclerosis. 3. The extent of correlation of degrees of atherosclerosis within and between the coronary and cerebral vascular beds. American Journal of Cardiology, 6, 300-308. 